\title{
IMPACTO DE LA ENFERMEDAD DE KAWASAKI EN LA POBLACIÓN PEDIÁTRICA
}

\author{
(IMPACT OF KAWASAKI DISEASE IN THE PEDIATRIC POPULATION)
}

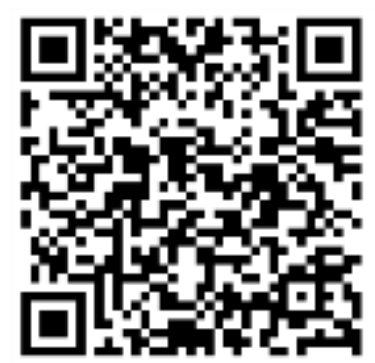

${ }^{1}$ Médico general, graduada de la Universidad de Ciencias Médicas (UCIMED), médico investigadora independiente. vale.molina06@gmail.com

${ }^{2}$ Médico general, graduado de la Universidad de Ciencias Médicas (UCIMED), médico investigador independiente. daniel.barquero95@hotmail.com

${ }^{3}$ Médico general, graduada de la Universidad de Costa Rica (UCR), médico investigadora independiente.

mariana.penam193@gmail.com

\author{
${ }^{1}$ Dra. Valeria Nicole Molina Jiménez \\ Investigadora independiente, San José, Costa Rica \\ vale.molina06@gmail.com \\ https://orcid.org/0000-0001-5359-9473 \\ ${ }^{2}$ Dr. Daniel Esteban Barquero Orias \\ Investigador independiente, San José, Costa Rica \\ daniel.barquero95@hotmail.com \\ (D) https://orcid.org/0000-0001-7627-1358 \\ ${ }^{3}$ Dra. Mariana Peña Miranda \\ Investigadora independiente, San José, Costa Rica \\ mariana.penam193@gmail.com \\ https://orcid.org/0000-0003-0753-7217 \\ RECIBIDO \\ CORREGIDO \\ ACEPTADO \\ $7 / 12 / 2018$ \\ $17 / 12 / 2018$ \\ $21 / 12 / 2018$
}

\section{RESUMEN}

La enfermedad de Kawasaki es una vasculitis aguda, autolimitada, actualmente de etiología desconocida caracterizada por una respuesta inflamatoria en pacientes genéticamente predispuestos a padecer la enfermedad. Afecta principalmente a niños menores de 5 años y su diagnóstico se realiza basado principalmente en las características clínicas y con apoyo de estudios de laboratorio o con la demostración de aneurismas coronarios. Esta patología es la principal causa de enfermedad cardiaca adquirida en la niñez, debido a que la vasculitis afecta a vasos de mediano calibre, principalmente las arterias coronarias, lo cual desencadena formación de aneurismas coronarios con posterior trombosis. Se debe de realizar un diagnóstico temprano para poder dar el tratamiento adecuado y evitar consecuencias fatales por el desarrollo de aneurismas coronarios, los cuales se desarrollan en un $25 \%$ de los pacientes que no reciben tratamiento.

PALABRAS CLAVES: síndrome mucocutáneo linfonodular, vasculitis, fiebre, exantema, aneurisma. 


\begin{abstract}
Kawasaki disease it's an acute vasculitis of unknown etiology that is characterized by an immune response in patients that are genetically predispose to develop the disease. It affects children below the age of 5 predominantly. The diagnosis is made based on the clinical characteristics, laboratory data and demonstration of coronary aneurisms with an echocardiography. This disease is the primary cause of acquired cardiac disease in children, since the vasculitis affects medium sized vessels, primarily the coronary arteries, which provoke the formation of coronary aneurisms that can trigger a thrombosis. It is essential to make an early diagnosis, so that the treatment can be established to prevent the fatal consequences of the disease product of the formation of aneurisms, which develop in $25 \%$ of patients that do no receive and adequate treatment.
\end{abstract}

KEYWORDS: Mucocutaneous lymph node syndrome, vasculitis, fever, exanthema, aneurysm.

\title{
INTRODUCCIÓN
}

La enfermedad de Kawasaki fue descrita por primera vez en 1964 por el Dr. Tamisaku Kawasaki en Japón. Inicialmente fue descrita como un síndrome mucocutánea linfático. Para este entonces, se desconocía la afectación cardiaca de la enfermedad(1). Y fue hasta en 1970 cuando se empezó a ver que pacientes con este síndrome morían de forma repentina por oclusiones trombóticas de las arterias coronarias (2). Fue cuando el Dr. Noboru Tanaka evidenció aneurismas y trombosis coronaria en autopsias de niños con esta enfermedad (3).

Se caracteriza por ser una vasculitis aguda y autolimitada, de etiología desconocida hasta el momento, la cual afecta predominantemente a infantes menores de 5 años, sin embargo se puede presentar en formas atípicas a cualquier edad (1).

No existe un examen diagnóstico específico para esta patología y el diagnóstico se hace basándose principalmente en las características clínicas, con el apoyo de los exámenes de laboratorio y de los estudios de gabinete como el ecocardiograma. Actualmente, la enfermedad de Kawasaki es la principal causa de enfermedad cardiaca adquirida en niños en países de desarrollo (1).

Aproximadamente un $25 \%$ de los pacientes que no reciben tratamiento llegan desarrollar aneurismas coronarios, porcentaje que se puede llegar a disminuir a un $5 \%$ con un tratamiento temprano (4). En esta revisión se planea hacer un resumen general de esta enfermedad, describiendo la epidemiología mundial, nuevos descubrimientos en la etiología, características clínicas, diagnóstico, manejo y pronóstico con el propósito de tener esta patología en mente en el abordaje del niño febril para así poder hacer un diagnóstico 
certero y un manejo adecuado con el fin de evitar las complicaciones a largo plazo.

\section{EPIDEMIOLOGÍA}

La enfermedad de Kawasaki afecta en aproximadamente un $80 \%$ a niños menores de 5 años, sin embargo esta enfermedad puede ocurrir incluso en la adolescencia. Se ha sugerido que la maduración del sistema inmune es lo que se ha asociado a que esta patología suceda a edades tan tempranas (1).

La incidencia de la enfermedad varía entre diferentes poblaciones. En Norteamérica, Australia y Europa tienen una incidencia actual de 4-25/100 000 niños menores de 5 años, con importantes diferencias entre los diferentes grupos étnicos en estas poblaciones. Particularmente, en Estados Unidos, existe una incidencia actual de 17.5-20.8/100 000, con tasas más altas entre personas Afroamericanas y de las Islas del Pacífico. La incidencia de americanos japoneses viviendo en Hawaii es similar a los de la población en Japón. Lo cual sugiere una importante contribución genética a la susceptibilidad de padecer la enfermedad de Kawasaki. En Europa se reportan incidencias de <16/ 100 000. En Japón y Korea, se reportan incidencias 1020 veces más altas que en EEUU y Europa, incidencia la cual sigue en aumento. En Japón, la incidencia actual se reporta en 265/ 100000 en < 5 años. La incidencia también sigue en aumento en China e India (4).

La Enfermedad de Kawasaki tiene estacionalidad, por ejemplo, en Estados Unidos la mayoría de casos reportados son en invierno. A diferencia de Japón que reporta los picos estacionales en Enero y Julio. Existen hipótesis acerca de la estacionalidad de la enfermedad. Entre ellas, se han hechos observaciones acerca de los cambios estacionales en los vientos troposféricos del norte del Pacífico se

correlacionan en forma importante con casos de enfermedad de Kawasaki en Hawaii, el sur de California y Japón. También se ha asociado con los vientos del norte del desierto en Chile. Se ha propuesto que la Enfermedad de Kawasaki puede ser desencadenada por agentes llevados por los vientos que se originan en Asia central. Muestreo del aire de la troposfera ha mostrado altas densidades de esporas de Candida albicans, sin embargo aún no está claro si esto es suficiente o necesario para causar la enfermedad de Kawasaki y se deben de realizar más estudios (4).

Se ha planteado que esta patología puede tener un trasfondo genético y es por esto que se han visto tasas de incidencia de 1.4$2.1 \%$ entre hermanos, tasas mucho más altas que en la población normal (5).

\section{FISIOPATOLOGÍA Y PATOGENIA}

A pesar de décadas de investigación, la etiología de esta enfermedad sigue siendo desconocida. Se han generado teorías acerca de virus respiratorios que ingresan a través del tracto respiratorio superior, desencadenando una respuesta inmune en pacientes genéticamente susceptibles (6). La respuesta inmune que se da ante la fase aguda de la Enfermedad de Kawasaki incluye la activación una respuesta inmune innata (por activación de receptores toll tipo 2 en los macrófagos en sangre periférica) y adaptativa (1). Los neutrófilos son los primeros en responder generando una invasión de la pared arterial; seguido de células T CD $8+$, células dendríticas y 
macrófagos/monocitos. Existe también una activación endotelial y de las células plasmáticas $\lg A$ oligoclonales, las cuales parecen estar involucrados en la formación de la arteritis coronaria (5). La infiltración de células plasmáticas $\lg \mathrm{A}$ en el tracto respiratorio, sugiere una puerta de entrada respiratoria por el agente o los agente etiológicos causantes de esta enfermedad. También, se ha planteado que enzimas como las metaloproteinasas de matriz, pueden infiltrar y dañar la integridad de la pared arterial contribuyendo a la formación de los aneurismas de las arterias coronarias. Se cree que también existe un importante aporte de la susceptibilidad genética en el desarrollo de la enfermedad y es por esto que se han visto patrones raciales y familiares. Los genes que más se han estudiados son el gen Inositol 1,4,5trifosfato 3-kinasa (ITPKC), gen caspasa-3 (CASP3) , Linfocito B kinasa (BLK), gen CD40, el receptor Fc del fragmento de baja afinidad lla de IgG (FC- GR2A), y HLA. Se realizaron estudios en Japón, lugar donde se han documentado la mayor cantidad de casos y se vio asociaciones con las regiones 12q24 y 19q13.2 (donde se encuentra el gen ITPKC). El gen ITPKC actúa como un contrarregulador de la activación de los células $T$ y es por esto que ante polimorfismos de este gen, habría un aumento en la activación de células $T$ lo que llevaría a un aumento en la expresión de citoquinas. Esto llevaría a un aumento en la susceptibilidad y severidad de la enfermedad (5).

Los cambios patológicos que se observan en la enfermedad de Kawasaki afectan principalmente arterias extra parenquimatosas, musculares y de mediano calibre, más frecuentemente las arterias coronarias. Se dan 3 procesos en la pared arterial: arteritis necrotizante, vasculitis aguda y subaguda y proliferación miofibroblástica luminal. La vasculitis aguda se caracteriza por una infiltración neutrofílica que se puede asociar a una necrosis extensa de todas las paredes del vaso. Las elastasas de los neutrófilos pueden atacar la lamina elástica interna y externa y contribuir a la formación de aneurismas. La vasculitis subaguda se puede observar semanas luego del inicio de la fiebre y persiste por meses a años y está más asociada con la proliferación miofibroblástica luminal, la cual puede resultar en estenosis de los vasos con posterior isquemia miocárdica (5).

\section{PRESENTACIÓN CLÍNICA}

Las principales características clínicas son las fiebre asociado a alteraciones mucocutáneas en extremidades, boca, adenopatía cervical y conjuntivitis bilateral no exudativa. La fiebre es generalmente alta, entre $39-40^{\circ} \mathrm{C}$ y remitente. La fiebre puede continuar por 1-3 semanas y usualmente resuelve 36 horas luego de la aplicación de la inmunoglobulina, en casos donde la fiebre no resuelve, se consideraría que el paciente es resistente a la inmunoglobulina y se deben de buscar otras terapias. Los cambios en las extremidades, como el eritema de las palmas y plantas con induraciones firmes y algunas veces dolorosas en las manos y los pies, ocurren en la fase aguda. Descamación de las manos y los pies ocurre principalmente 2-3 semanas luego del inicio de la fiebre e inicia principalmente en la zona periungueal. El brote eritematoso aparece, usualmente 5 días luego del inicio de la fiebre y se caracteriza por ser un maculopapular difuso. También, puede aparecer en otras 
formas como un eritrodermia escarlatiniforme, un brote similar al eritema multiforme y otras formas menos comunes son: brote urticarial o una erupción fina micropustular (6). El brote usualmente involucra tronco y extremidades, con acentuación en la región perianal a lo cual se le asocia descamación temprana (7). En caso de tener brotes bulosos, vesiculares o petequiales, se deben buscar otros diagnósticos. La conjuntivitis bulbar, bilateral y no exudativa usualmente inicia luego del inicio de la fiebre y usualmente respeta el limbo esclerocorneal. Se puede observar una uveítis anterior durante la primera semana de la fiebre. Otros hallazgos son, hemorragia subconjuntival y queratitis punteada. Los cambios orales son eritema, resequedad, fisuras y sangrado de los labios, lengua en fresa con eritema y papilas fungiformes prominente y eritema difuso de la mucosa orofaríngea. Cambios como úlceras orales o exudados faríngeos no son consistentes con este enfermedad. La linfadenopatía cervical es la característica menos frecuente entre las principales. Este hallazgo usualmente es unilateral, $\geq 1.5 \mathrm{c}$, de diámetro en el triángulo cervical anterior y se debe de hacer diagnóstico diferencial con una linfadenitis bacteriana. En la enfermedad de Kawasaki, es usual que varios nódulos linfáticos estén agrandados, en la linfadenitis bacteriana no (6). Existen pacientes que se presentan con inestabilidad hemodinámica en la fase aguda, denominado síndrome de shock de la enfermedad de Kawasaki y la causa aún no se ha definido (8).

La enfermedad de Kawasaki puede tener otros hallazgos clínicos en los diferentes sistemas del cuerpo. En el sistema cardiovascular se puede observar miocarditis, pericarditis, insuficiencia valvular, shock, alteraciones de las arterias coronarias, aneurismas de arterias de mediano calibre, gangrena periférica, agrandamiento de la raíz aórtica. En el sistema respiratorio, se pueden encontrar infiltrados intersticiales y peribronquiales en la radiografía de tórax y nódulos pulmonares. En el sistema musculoesquelético puede haber artritis y artralgias. Se puede observar pleocitosis en los análisis del líquido sinovial. En el sistema gastrointestinal, diarrea, vómitos, dolor abdominal, hepatitis, ictericia, hidrops vesicular y pancreatitis. En el sistema nervioso, los pacientes pueden presentar irritabilidad, meningitis aséptica con pleocitosis en el líquido cefalorraquídeo, parálisis facial y sordera neurosensorial. En el sistema genitourinario, uretritis e hidrocele. $Y$ otros hallazgos de importancia son el eritema e induración del sitio de inyección de la BCG (7).

\section{DIAGNÓSTICO}

El diagnóstico de Enfermedad de Kawasaki clásico se hace basado en la presencia de $\geq 5$ días de fiebre y la presencia de $\geq 4$ de 5 de las características clínicas principales, las cuales son: cambios a nivel oral (eritema o labios agrietados, lengua en fresa $y / 0$ eritema de la mucosa oral 0 faríngea), conjuntivitis bulbar bilateral sin exudados, brote (maculopapular, eritrodermia difuso o simular al eritema multiforme), alteración en extremidades (eritema y edema de las manos y pies en la fase aguda o descamación periungueal en la fase subaguda) y adenopatía cervical con un diámetro $\geq 1.5 \mathrm{~cm}$, usualmente unilateral. Para hacer el diagnóstico se debe de tener más de 4 de los criterios clínicos principales, particularmente cuando está presente el edema y eritema de manos y pies (6). 
Se debe de considerar el diagnóstico de enfermedad de Kawasaki incompleto o atípico en los pacientes que tengan fiebre prolongada e inexplicable y alguna de las características clínicas principales. diagnóstico se confirmaría en caso de que se observen aneurismas coronarios en la ecocardiografía (6). Las consecuencias cardiovasculares ocurren por igual en la enfermedad completa e incompleta (7).

\section{ESTUDIOS COMPLEMENTARIOS}

Entre los principales hallazgos en los laboratorios se encuentra leucocitosis durante la fase aguda de la enfermedad con predominio de granulocitos maduros e inmaduros. Casos donde hay leucopenia o predominancia linfocítica sugieren diagnósticos alternativos. Puede haber anemia, usualmente es normocíticanormocrómica y resuelve con la resolución de la inflamación. Hay elevación de los reactantes de fase aguda como la VES y la PCR. No se debería de utilizar la VES para evaluación de la evolución debido a que ésta aumenta tras la administración de inmunoglobulina intravenosa, en este caso de PCR es más útil como marcador de inflamación luego del tratamiento. La trombocitosis es algo característico de la enfermedad de Kawasaki, sin embargo no ocurre hasta la segunda semana con un pico en la tercera semana, normalizándose en la cuarta a sexta semana luego del inicio de los síntomas. Puede ocurrir trombocitopenia en las primeras dos semanas de la enfermedad, esta puede ocurrir en el contexto de una coagulación intravascular diseminada y es un factor de riesgo para el desarrollo de anormalidades cardiacas. Pueden haber elevaciones leves a moderadas de las transaminasas y de la
GGT en un $40-60 \%$ de los pacientes. La hipoalbuminemia es común y está asociada a enfermedad aguda más severa y prolongada. El examen general de orina puede mostrar piuria en $80 \%$ de los pacientes. Los análisis de líquido cefalorraquídeo pueden mostrar pleocitosis con predominio de células mononucleares, niveles de glucosa y proteínas normales. El pro-BNP puede estar elevado cuando hay daño miocárdico (6).

La ecocardiografía es el estudio primario para valorar el corazón. El primer ecocardiograma se debería de realizar tan pronto como se sospeche el diagnóstico, sin embargo nunca se debe de retrasar el inicio del tratamiento por hacer el diagnóstico, en casos donde no se pueda realizar 0 el primer ecocardiograma realizado no sea de buena calidad (porque el niño no estaba sedado), entonces se debe de realizar dentro de las 48 horas luego de haber hecho el diagnóstico e iniciado el tratamiento. Según las recomendaciones de la American Heart Association (AHA), en pacientes sin complicaciones se debe de realizar otro ecocardiograma 1-2 semanas después del primero, durante el tratamiento y luego 4-6 semanas después del tratamiento. Pacientes con importante afectación coronaria, detectados durante la fase aguda de la enfermedad, se deben de realizar ecocardiogramas más seguidos (al menos 2 por semana) hasta que las dimensiones luminales de los vasos coronarios hayan parado de progresar para así poder determinar el riesgo de trombosis (6).

\section{DIAGNÓSTICO DIFERENCIAL}

Entre los principales diagnósticos diferenciales se encuentran el sarampión, 
otras infecciones virales como el adenovirus o los enterovirus, fiebre escarlatina, síndrome de Stevens Johnson, leptospirosis, entre otros (6). No se debe de descartar la enfermedad de Kawasaki en pacientes que tienen clínica sugestiva de esto pero que al mismo tiempo tienen PCR positivas por virus, incluyendo el adenovirus $\mathrm{u}$ otros virus respiratorios (9).

\section{ENFERMEDAD DE KAWASAKI EN MENORES DE 6 MESES}

Pacientes diagnosticados con enfermedad de Kawasaki a edades tan tempranas, usualmente tienen formas atípicas. Se pueden presentar incluso con convulsiones y nefritis (10).

Los principales cambios observados fueron a nivel de extremidades, mucosa oral y el brote, sin embargo la linfadenopatía se presenta en pocos casos. La inflamación del sitio de inyección de la vacuna BCG es una característica frecuente en niños de esta edad.

En niños menores de 3 meses, el diagnóstico puede ser incluso más complejo debido a que muchas veces el diagnóstico está acompañado de infecciones por virus respiratorios, vómitos o diarrea (11). En un estudio realizado en China, se observó que un $80 \%$ de los niños diagnosticados con enfermedad de Kawasaki, menores de 3 meses, tenían anormalidades en las arterias coronarias, una mayor incidencia comparado con niños mayores (11).

Es muy importante tener la enfermedad de Kawasaki entre los diagnósticos diferenciales en niños con fiebre sin un origen claro, especialmente en menores de 6 meses, debido a que la mayoría de casos son atípicos con manifestaciones clínicas incompletas y tienen diagnósticos más tardíos, lo que resulta en mayor afección de las arterias coronarias, principalmente formación de aneurismas (10).

\section{MANEJO}

En el manejo del paciente con enfermedad de Kawasaki, es importante determinar ciertos factores de riesgo que pueden predisponer al paciente a tener un cuadro más severo y con esto mayor riesgo de desarrollar lesiones a nivel cardiaco. Dentro de los factores de riesgo que se han propuesto para esto son mayor edad al momento del diagnóstico, niveles altos de leucocitos, PCR, plaquetas y niveles bajos de albúmina. Esto implicaría que mayores niveles de inflamación están asociados con daño cardiaco (12).

La meta de la terapia en la fase aguda es reducir la inflamación y con esto el daño arterial para prevenir trombosis en aquellos con alteraciones coronarias (6).

tratamiento inicia con la inmunoglobulina intravenosa, la cual se debe de iniciar en los primeros 10 días desde el inicio de la fiebre (día 1). En caso de niños que se presentan luego de 10 días de sintomatología en caso de que persistan con inflamación sistémica, marcada por elevación de VES y PCR además de fiebre persistente sin otra explicación 0 aneurismas coronarios. En caso de que los laboratorios hayan normalizado y el ecocardiograma se reporte sin alteraciones, no es necesario aplicar la inmunoglobulina. En caso de que sea una enfermedad de Kawasaki recurrente, se debe de instaurar la terapia como inmunoglobulina intravenosa y aspirina (6).

La inmunoglobulina intravenosa (IGIV), administrada en forma temprana, reduce la incidencia de anomalías coronarias (13). Se cree que por una modulación en la 
producción de citoquinas, neutralización de toxinas y otros agentes patógenos, aumento de la actividad reguladora de células $T$, supresión de la síntesis de anticuerpos y provisión de anticuerpos antiidiotípicos. La dosis estándar es $2 \mathrm{~g} / \mathrm{kg}$ en infusión, usualmente a pasar en 10-12 horas, junto con ácido acetilsalicílico (6). No se puede vacunar a los pacientes contra sarampión, rubeola ni varicela en los próximos 11 meses luego de aplicada la IGIV (6).

El uso de ácido acetilsalicílico (AAS) se utiliza en conjunto con la inmunoglobulina intravenosa, a pesar de su actividad antiinflamatoria a dosis altas, esta no disminuye el riesgo de desarrollo de anomalías coronarias (6). Durante la fase aguda, el ácido acetilsalicílico se administra a dosis de $80-100 \mathrm{mg} / \mathrm{kg} /$ día cada 6 horas en EEUU y a dosis de $30-50 \mathrm{mg} / \mathrm{kg} /$ día cada 6 horas en Japón y Europa. Se debe disminuir la dosis, luego de que el paciente se encuentre afebril durante 48 a 72 horas. La dosis a la cual se modifica es de 3$5 \mathrm{mg} / \mathrm{kg} /$ día y se continua por 6-8 semanas desde el inicio de los síntomas. En el caso de niños en los cuales se evidencian aneurismas coronarios, el AAS se puede continuar de forma indefinida (6).

Se pueden utilizar en conjunto con la IGIV y el AAS, los corticoesteroides. Se han evaluado regímenes con prednisolona y se ha observado que se disminuye la duración de la fiebre y la prevalencia de aneurismas coronarios (6). Se ha propuesto la combinación de la dosis estándar de IGIV más el uso de esteroides como parte del manejo inicial en pacientes con diagnóstico de enfermedad de Kawasaki de alto riesgo, para disminuir la tasa de anormalidades de las arterias coronarias, sin embargo la AHA no lo recomienda como terapia primaria de rutina (6).

Existe un $10-20 \%$ de pacientes los cuales se denominan resistentes a la inmunoglobulina intravenosa, los cuales persisten con fiebre luego de 36 horas de aplicada la IGIV. En estos casos se puede aplicar una segunda dosis de IGIV a la dosis recomendada, como por ejemplo metilprednisolona $20-30 \mathrm{mg} / \mathrm{kg} /$ día IV por 3 días o incluso por 2-3 semanas. Otra opción es la administración de Infliximab (5 mg/kg), un anticuerpo monoclonal contra el factor de necrosis tumoral alfa ya que se ha documentado que en la enfermedad de Kawasaki existen niveles elevados de esta citoquina proinflamatoria, sobre todo en pacientes que desarrollaron anomalías coronarias. Se podría considerar también el uso de Ciclosporina en pacientes que no responden a la segunda dosis de IGIV, esteroides ni infliximab. Siempre, se debe continuar el tratamiento con el AAS. Se debe de hacer un seguimiento de cerca a los pacientes con enfermedad de Kawasaki resistente o refractaria (14).

\section{COMPLICACIONES}

Los pacientes con enfermedad de Kawasaki desarrollan una serie de complicaciones. La incidencia de aneurismas coronarios gigantes es de 1-4\% y la ecocardiografía es el principal método para el diagnóstico de estos (15). Éstos pacientes, tienen un alto riesgo de trombosis y es por esto que el tratamiento con AAS se debe de mantener a dosis de 3-5mg/kg/día hasta 4-6 semanas luego del inicio de la enfermedad. Pacientes con aneurismas coronarios que se expanden rápidamente se debe de iniciar terapia de anticoagulación con heparinas de bajo peso molecular o warfarina, además 
del tratamiento con AAS. Pacientes con alto riesgo de trombosis con aneurismas coronarios gigantes, se debe de dar terapia triple con doble antiagregación plaquetaria además de anticoagulación (6). Se dice que los pacientes con aneurismas coronarios gigantes tienen el peor pronóstico, por el alto riesgo de desarrollar trombosis coronaria, estenosis o infarto de miocardio, con el mayor riesgo de infarto agudo al miocardio en el primer año desde el inicio de la enfermedad (3). Existen también otras complicaciones como anormalidades miocárdicas, por infarto agudo al miocardio, arritmias, anormalidades en la aorta, insuficiencia valvular, entre otros (6).

\section{RIESGO Y SEGUIMIENTO}

Pacientes con enfermedad de Kawasaki son estratificados en varios grupos de riesgo dependiendo del grado de afectación coronaria. Factores que se deben de tener en cuenta son la longitud del aneurisma y si está localizado distalmente, si hay ausencia de colaterales o estas están obstruidas, antecedentes de trombosis, infarto agudo al miocardio, revascularización o presencia de disfunción ventricular. El seguimiento depende de estos factores y esto se evalúa luego de la fase aguda (13). Pacientes sin secuelas cardiacas conocidas durante el primer mes del inicio de la enfermedad de Kawasaki, usualmente vuelven a su estado basal previo a la patología con un excelente estado de salud (16).

Las lesiones coronarias desarrolladas en pacientes con Enfermedad de Kawasaki tienen cambios dinámicos en el tiempo. Existe resolución angiográfica de los aneurismas en un $50-67 \%$ de los pacientes luego de 1-2 años de resolución de la enfermedad (6). En pacientes donde nunca se documentó cambios a nivel coronario o bien tuvieron resolución de estos se les puede dar de alta, siempre teniendo en cuenta factores de riesgo cardiovascular (13).

La mortalidad en pacientes con secuelas a nivel cardiaco luego de esta patología es mayor que en la población normal. Se han documentado una alta prevalencia de secuelas cardiacas relacionados con enfermedad de Kawasaki en adultos jóvenes. En EE.UU, 5\% de los pacientes con infarto agudo al miocardio, menores de 40 años ocurren en pacientes con historia conocida o sospechada de enfermedad de Kawasaki (13).

\section{CONCLUSIONES}

La enfermedad de Kawasaki es una vasculitis de vasos de mediano calibre, principalmente las arterias coronarias, autolimitada, la cual, hasta el día de hoy se desconoce su etiología. Se plantean varias teorías sobre una respuesta inmune y adaptativa ante un agente que podría ser un virus respiratorio y otro en sujetos genéticamente predispuestos.

El diagnóstico se hace basado en las características clínicas principales, sin embargo existen formas atípicas 0 incompletas de la enfermedad que deben de ser tomadas en cuenta debido a que usualmente se hacen diagnósticos más tardíos con las consecuencias cardiacas importantes que tiene la enfermedad. Esta patología es la principal causa de enfermedad cardiaca adquirida en niños, en países en desarrollo y ahí radica su importancia. Por lo tanto se concluye que en el manejo de un niño febril, sin foco claro y con las características antes mencionadas, siempre se debe de tener en cuenta esta patología como parte de los 
IMPACTO DE LA ENFERMEDAD DE KAWASAKI EN LA POBLACIÓN PEDIÁTRICA - Dra. Valeria Nicole Molina Jiménez Dr. Daniel Esteban Barquero Orias / Dra. Mariana Peña Miranda doi: $\underline{\text { https://doi.org/10.31434/rms.v4i1.201 }}$

diagnósticos diferenciales, incluso en presencia de otros virus respiratorios, para así poder hacer un diagnóstico temprano e instaurar la terapia en la fase aguda (primeros 10 días desde el inicio de la fiebre) y así poder disminuir de un $25 \%$ a un
$5 \%$ la incidencia de alteraciones coronarias, principalmente la formación de aneurismas coronarios y con esto las consecuencias a largo plazo que se presentan.

\section{REFERENCIAS}

1. Newburger J, Takahashi M, Burns J. Kawasaki Disease. Journal of the American College of Cardiology. 2016; 67(14): 1738-1749. https://doi.org/10.1016/j.jacc.2015.12.073

2. Gámez L, Yamazaki M. Enfermedad de Kawasaki: un vistazo al cincuenteno. Revista Investigación Médica Sur de México. 2015; 22(4): 169-181. Disponible en:https://www.medigraphic.com/pdfs/medsur/ms-2015/ms154c.pdf

3. Matiz Mejía S, Ariza Correa C, Salinas Suárez C, Huertas Quiñones M, Sanguino Lobo R. Enfermedad de Kawasaki. Revista Colombiana de Cardiología. 2017; 24(3): 307.e1307.e6.https://doi.org/10.1016/j.rccar.2016.05.011

4. Singh S, Vignesh P, Burgner D. The epidemiology of Kawasaki disease: a global update. Archives of Disease in Childhood. 2015; 100(11): 1084-1088. https://doi.org/10.1136/archdischild-2014-307536

5. Kim K, Kim D. Recent Advances in Kawasaki Disease. Yonsei Medical Journal. 2016; 57(1): 1521. https://doi.org/10.3349/ymi.2016.57.1.15

6. McCrindle B, Rowley A, Newburger J, Burns J, Bolger A, Gewitz M et al. Diagnosis, Treatment, and Long-Term Management of Kawasaki Disease: A Scientific Statement for Health Professionals From the American Heart Association. Circulation. 2017; 135(17): e927e999.https://doi.org/10.1161/CIR.0000000000000484

7. Gil G, Nieto N, León A, Hernandez Z. Enfermedad de Kawasaki. Revista Hospital Juárez de México. 2018; 85(3): 154-158. Disponible en: https://www.medigraphic.com/pdfs/juarez/ju-2018/ju183f.pdf

8. Chen P, Chi H, Huang F, Peng C, Chen M, Chiu N. Clinical manifestations of Kawasaki disease shock syndrome: A case-control study. Journal of Microbiology, Immunology and Infection. 2015; 48(1): 43-50. https://doi.org/10.1016/j.jmii.2013.06.005

9. Turnier J, Anderson M, Heizer H, Jone P, Glode M, Dominguez S. Concurrent Respiratory Viruses and Kawasaki Disease. PEDIATRICS. 2015; 136(3): e609-e614. https://doi.org/10.1542/peds.2015$\underline{0950}$

10. Singh S, Agarwal S, Bhattad S, Gupta A, Suri D, Rawat A et al. Kawasaki disease in infants below 6 months: a clinical conundrum?. International Journal of Rheumatic Diseases. 2016; 19(9): 924928.https://doi.org/10.1111/1756-185X.12854 
11. Li W, Zhang L, Huang $P$, Zhang Z. Clinical features and mid-term follow-up in infants younger than 3 months with Kawasaki disease in a Chinese population. Journal of Paediatrics and Child Health. 2018; e14233. https://doi.org/10.1111/jpc.14233

12. Kuwabara M, Yashiro M, Kotani K, Tsuboi S, Ae R, Nakamura $Y$ et al. Cardiac Lesions and Initial Laboratory Data in Kawasaki Disease: a Nationwide Survey in Japan. Journal of Epidemiology. 2015; 25(3) :189-193. https://doi.org/10.2188/jea.JE20140128

13. Barrios Tascón A, Centeno Malfaz F, Rojo Sombrero H, Fernández-Cooke E, Sánchez-Manubens J, Pérez-Lescure Picarzo J. Consenso nacional sobre diagnóstico, tratamiento y seguimiento cardiológico de la enfermedad de Kawasaki. Anales de Pediatría. 2018; 89(3): 188.e1188.e22.https://doi.org/10.1016/j.anpedi.2018.04.003

14. Owens S, Agyeman P, Whyte M, Crossland D, Flood T, Abinun M. Aggressive anti-inflammatory treatment for refractory Kawasaki disease. Journal of Infection. 2017; 74(1): 9195.https://doi.org/10.1016/i.jinf.2016.08.015

15. Sánchez Andrés A, Salvador Mercader I, Seller Moya J, Carrasco Moreno J. Giant coronary aneurysms in infants with Kawasaki disease. Anales de Pediatría. 2017; 87(2): 6572.https://doi.org/10.1016/j.anpede.2016.07.005

16. Kourtidou S, Slee A, Bruce M, Wren H, Mangione-Smith R, Portman M. Kawasaki Disease Substantially Impacts Health-Related Quality of Life. The Journal of Pediatrics. 2018; 193: 155163.e5.https://doi.org/10.1016/j.jpeds.2017.09.070 